\title{
Automatic Coastline Detection Using Image Enhancement and Segmentation Algorithms
}

\author{
Erdem Emin Maraş ${ }^{1 *}$, Mustafa Caniberk ${ }^{2}$, Hadi Hakan Maraş ${ }^{3}$ \\ ${ }^{1}$ Department of Geomatics, Ondokuz Mayıs University, Samsun, Turkey \\ ${ }^{2}$ Department of Photogrammetry, General Command of Mapping, Ankara, Turkey \\ ${ }^{3}$ Department of Computer Engineering, Cankaya University, Ankara, Turkey
}

Received: 29 February 2016

Accepted: 11 July 2016

\begin{abstract}
Coastlines have hosted numerous civilizations since the earliest times of mankind due to the advantages they offer such as natural resources, transportation, arable areas, seafood, trade, and biodiversity. Coastal regions should be monitored vigilantly by planners and control mechanisms, and any changes in these regions should be detected with its human or natural origin, and future plans and possible interventions should be formed in these aspects to maintain ecological balance, sustainable development, and planned urbanization.

Integrated coastal zone management (ICZM) provides an important tool to reach that goal. One of the important elements of ICZM is the detection of coastlines. While there are several methods to detect coastlines, remote sensing methods provide the fastest and the most efficient solutions.

In this study, color infrared, grayscale, RGB, and fake infrared images were processed with the median filtering and segmentation software developed within the study, and coastal lines were detected by the edge detection method. The results show that segmentation with fake infrared images derived from RGB images give the best results.
\end{abstract}

Keywords: coastlines, segmentation, edge detection, integrated coastal zone management (ICZM)

\section{Introduction}

Coastlines are environmental regions most subject to natural and artificial changes. Since coastlines lie at the intersection of land and sea, many natural phenomena have an impact on them. Moreover, the use of coastlines throughout history for settling, sheltering, and economic and transportation purposes has caused artificial changes to them [1]. Between 1807 and 1927, all coastlines

*e-mail:eemaras@omu.edu.tr were produced by terrestrial measurements. Aerial photogrammetry has been used to detect coastlines since 1927.

Aerial photogrammetry has been seen the sole means of detecting coastlines, but its use has always been expensive and time-consuming. Additionally, it requires extensive terrestrial work to distinguish coastlines on monochrome aerial photos when water is turbid and muddy.

The analog form of aerial photos makes it difficult to extract features and is error-prone. In 1972, LANDSAT started to transfer digital images in the infrared band in which land and water can be distinguished clearly. This led to real-time imaging of larger areas at once, and 
detailed feature extraction from the images of high spectral resolution [2]. With these developments in remote sensing imaging systems, different methodologies have been proposed to detect coastlines [3-7]. One of these methods is the combination of various bands and the automatic detection of coastlines by segmentation algorithms.

\section{Problem Definition}

Integrated coastal zone management (ICZM) is a dynamic, continuous, and iterative process and aims to support and strengthen sustainable management [8]. ICZM, which involves the complete cycle of data collection, planning, decision making, management, and monitoring of the application, and aims to take the necessary actions by using the participation and the cooperation of the shareholders. ICZM also tries to balance environmental, economic, social, cultural, and recreational objectives [9].

Coastal management is usually exercised to solve problems arising from the use of coastal zones. Several regulations and bylaws have been developed by several countries and international organizations (the United Nations Department of International Economic and Social Affairs, 1982; UNEP, 1995; IWICM, 1996; and the Organization for Economic Co-operation and Development) to define problems and coordinate possible solutions [10].

The starting point for ICZM is to determine the coastal lines unambiguously. The most important step in the process is to determine the coast lines as quickly and accurately as possible. At this point, automatic determination of the coast lines will both speed up the management process and help make better decisions.

\section{Median Filtering in Image Processing}

Median filtering is one method used in image and signal processing applications to remove noise. The main idea is to remove the large intensity variations by taking the median of the intensity values within a certain window. In other words, the filter enables us to detect and clean pixels that are distinctly different from neighboring ones [11].

The median filter differs from weighted mean filters in one particular aspect. The weighted mean filters find the weighted mean of the neighbors, and take another mean of the result with the original. On the other hand, the median filter sorts the values first and uses their median directly as a result. To obtain the median value, an odd number of neighboring pixels is usually chosen. If an even number of neighbors is used, then the average of two pixels in the middle is taken. Here, represents the neighbors of the point at $[\mathrm{m}, \mathrm{n}]$.

$$
y[m, n]=\operatorname{median}\{x[i, j],(i, j) \in w\}
$$

Median filter is highly useful to clean point and line noises without breaking the spatial continuity and $\left[\begin{array}{lll}25 & 28 & 34 \\ 45 & 41 & 56 \\ 38 & 46 & 29\end{array}\right] \Rightarrow\{25,28,29,34,38,41,45,46,56\}$

Fig. 1. The application of the median filter.

resolution. In this respect, while it is efficient in binary images, it is not practical in Gaussian noises. If the number of noisy pixels is equal to or larger than the neighboring pixels the filter is not efficient [12].

As seen in Fig. 1, the median filter takes the median of the values of the neighboring pixels after sorting them. For an image of size $3 \times 3$, the median value is the fifth value in the list of the sorted values.

\section{Image Segmentation}

Segmentation is the first and the most important step in object-based image processing. In the large sense, the segmentation is the process of obtaining objects by grouping the homogenous neighboring pixels based on the selected scale and homogeneity. The main objective in the process is to detect significant objects and obtain their geometries. The segmentation process minimizes the heterogeneity and the smallest unit in the image - not the pixels - becomes the objects.

One of the advantages that the segmentation process offers is to allow relatively few and spectrally homogenous objects instead of millions of pixels. Since segmentation is object-based analysis, the errors arising from the segmentation process affect all subsequent steps, including classification accuracy. In this respect, segmentation quality should be of outmost quality [12].

The efficiency of the general segmentation algorithm depends on the homogeneity concept. The scale parameter is used to determine the average object size. By using different homogeneity criteria, and spectral and geometrical information, the objects are improved and compared [13]. The size and the homogeneity of the objects differs in the segmentation process depending on the chosen parameters. The scale parameter and the geometry and integrity parameters included in the homogeneity criterion are applied in multi-resolution segmentation.

These segmentation parameters are user-defined and are usually determined using trial and error. Besides those parameters, giving different weighting coefficients to each image band causes tiny changes in the formation of the objects. The more weight given to a specific band, the more the pixel information in that band will be taken into account [14].

\section{Morphological Edge Detection}

Since detection of the object requires determining the boundaries, edge detection has been one of the most important steps of image processing. Since the marked transitions correspond to the object boundaries, most edge detection algorithms are based on those transitions. Prewitt 


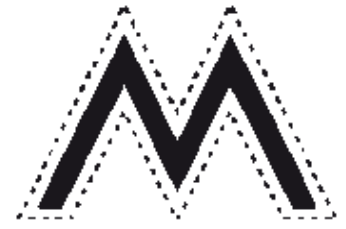

a) Erosion

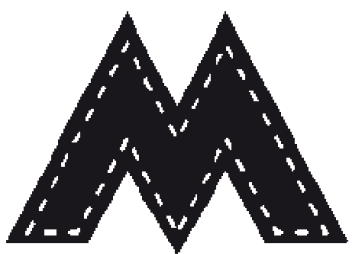

b) Dilation
Fig. 2. Sketch of the most fundamental morphological operators.

[15], Sobel [16], Canny [17], and Marr and Hilldreth [18] algorithms are usually acknowledged as the fundamental algorithms in the literature. Numerous studies employ these algorithms on grayscale images [19-21].

Mathematical morphology is an analysis and processing method based on set theory, topology, and random functions. This deals with geometric structures. Mathematical morphology is usually used in digital images. Besides, it is also used in graphics, surface joining, and various spatial structures [22-23]. The topological and geometrical concepts such as continuity and gaps characterize the mathematical morphology along with properties like shape, separation, integrity, and distance. Mathematical morphology is used to distinguish objects from other objects in the image. Moreover, it processes the objects in the images without changing their sizes. Mathematical morphology is first developed for binary images and further developed for grayscale images [2223].

The main idea behind morphology is to spatially correlate a pre-determined group of pixels on the image to detect the matching portions. These pixel groups are called "structuring element." The structuring element itself is a binary or grayscale image. The most fundamental morphological operators are erosion and dilation.

The object gets larger or thicker with dilation. On the contrary, when erosion is applied, the object shrinks and gets thinner. The effect of the operators depends on the structure and the size of the structuring element. With a

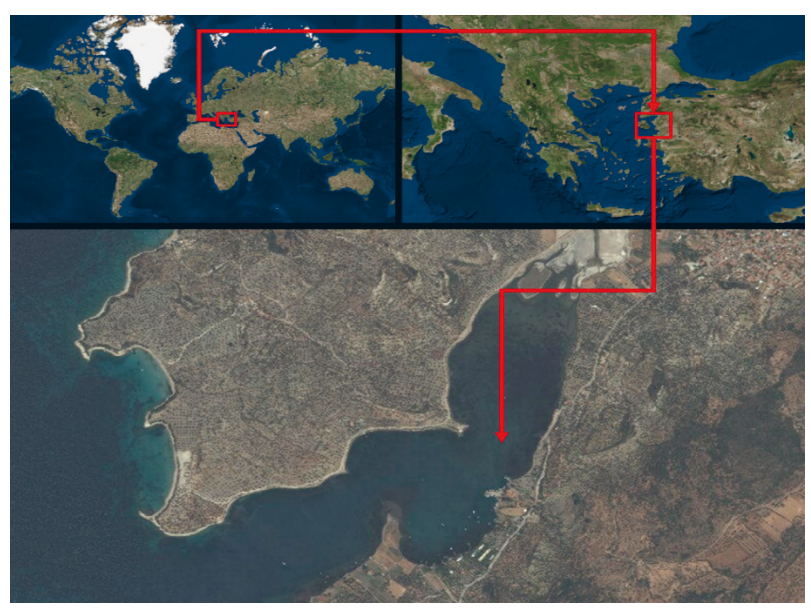

Fig. 3. Study area.

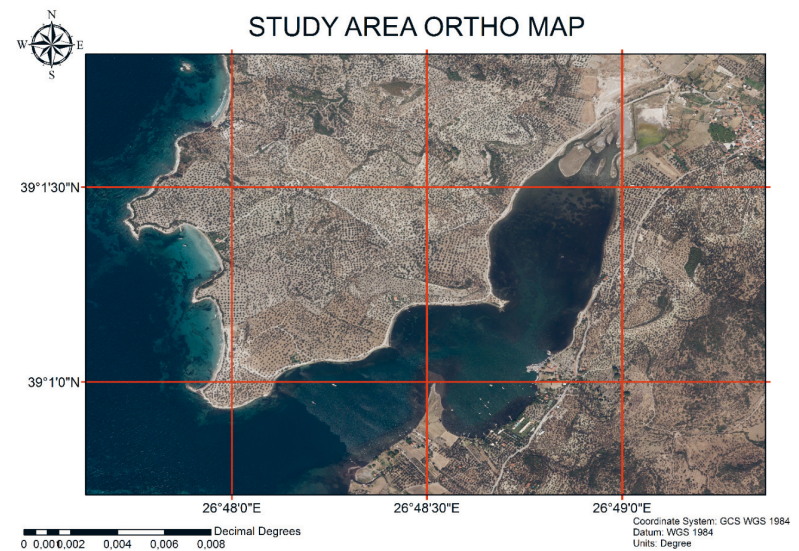

Fig. 4. Map produced from an aerial image with a resolution of $30 \mathrm{~cm}$.

sequential usage of erosion and dilation, higher-level operators like opening and closing can be formed.

\section{Aim of the Study}

Determining coastlines is a top priority for managing coastal zones. In this study, an automatic extraction of the coastal is presented by using the different bands of the remotely sensed images.

The edge detection in RGB images is more efficient than monochrome images [24]. The edge between a tow object with the same intensity but different color can be distinguished in color images. Thus, additional properties that go undetected in grayscale images can be identified in color images. As pointed out in [24], the results in color images are always better than those obtained in grayscale.

This approach, while it could be valid in various edgedetection studies, may not be suitable for use in coastal zone detection. In this study, the automatic detection of the coastlines is applied from the fake infrared images derived from easily accessible RGB images.

\section{Material and Methods}

The study area takes place on a coastal zone between the latitudes $39^{\circ} 00^{\prime} 43.8232^{\prime \prime} \mathrm{N}$ and $39^{\circ} 01^{\prime} 41.2731^{\prime \prime} \mathrm{N}$, and longitudes $26^{\circ} 47^{\prime} 59.9083$ 'E and 26 $6^{\circ} 49^{\prime} 10.6333^{\prime \prime} \mathrm{E}$ (Fig. 3).

The research material consists of orthorectified images obtained from aerial photos with a spatial resolution

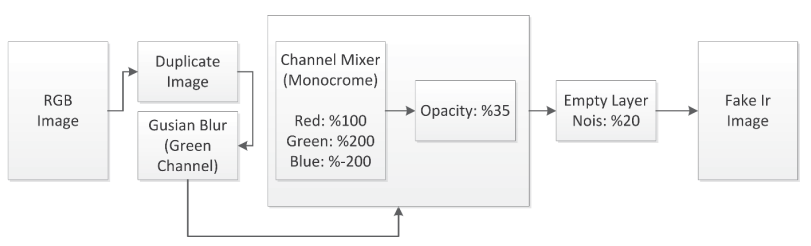

Fig. 5. Flow chart for producing a fake infrared image. 
a)

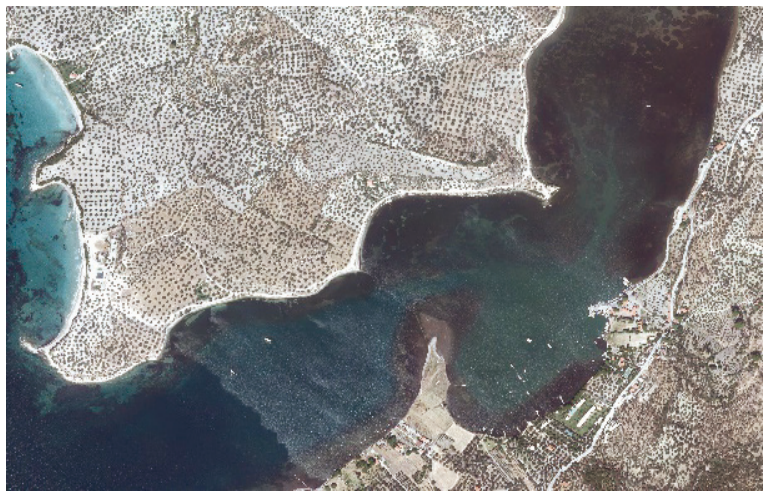

c)

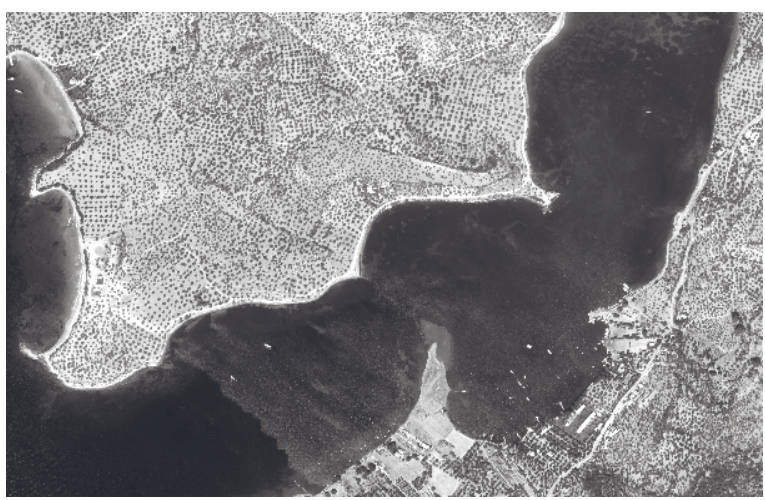

b)

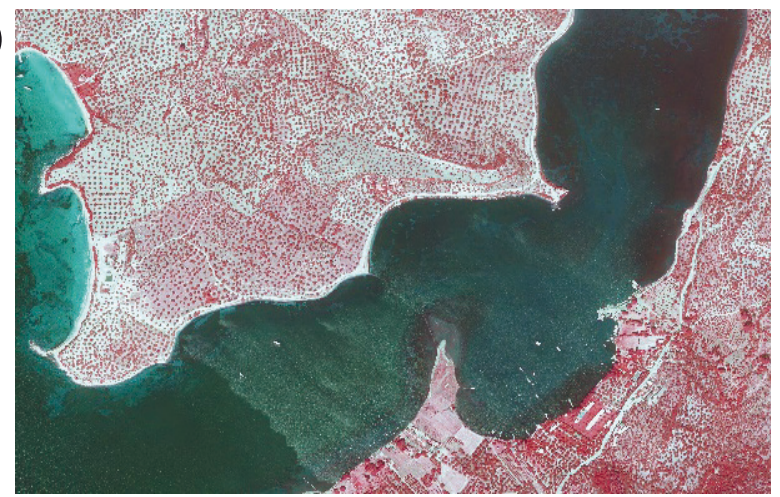

d)

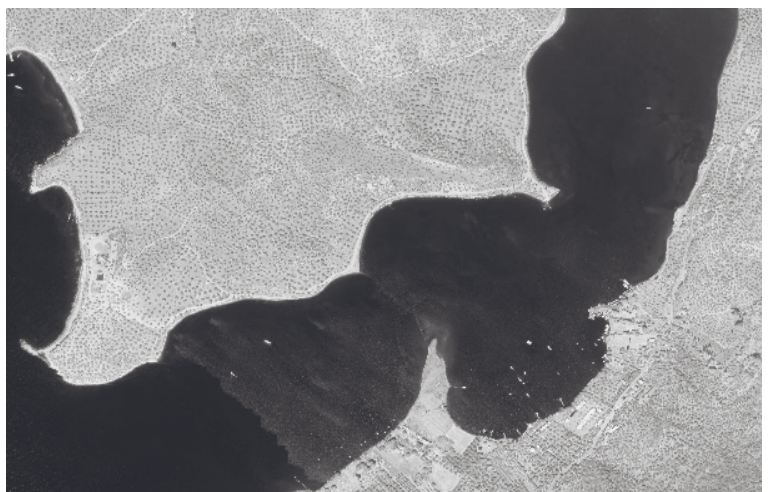

Fig. 6. The a) RGB, b) color infrared, c) grayscale, and d) fake infrared images of the study area.

of $30 \mathrm{~cm}$. The orthorectified images have both RGB and near-infrared bands (Fig. 4).

\section{Analysis}

In the first step, RGB, color infrared, grayscale fake infrared images were produced from four band images.
We used GlobalMapper software to prepare the images to be used in various band combinations. To produce fake infrared images from RGB images we used Photoshop software. After duplicating the RGB image, a Gaussian blur effect was applied in the green band and a "channel mixer" layer was added. The edges in infrared images seem to gloss with an eerie inner light, getting the

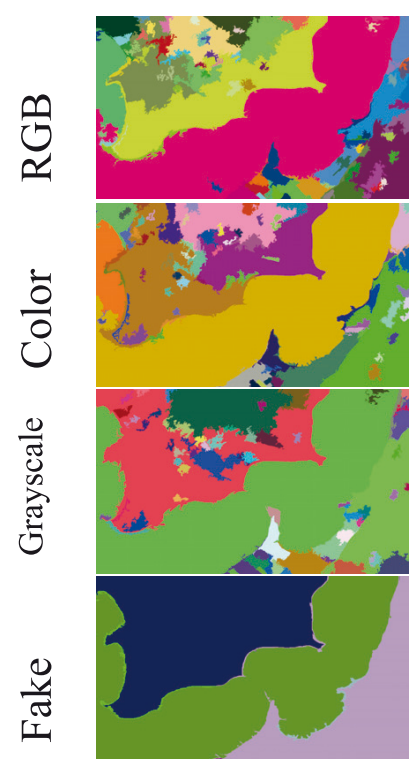

a)

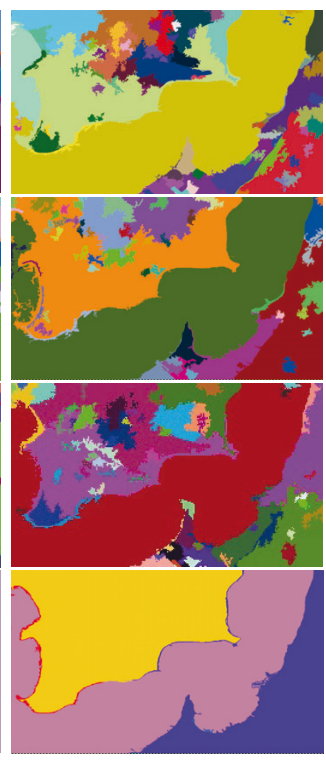

b)

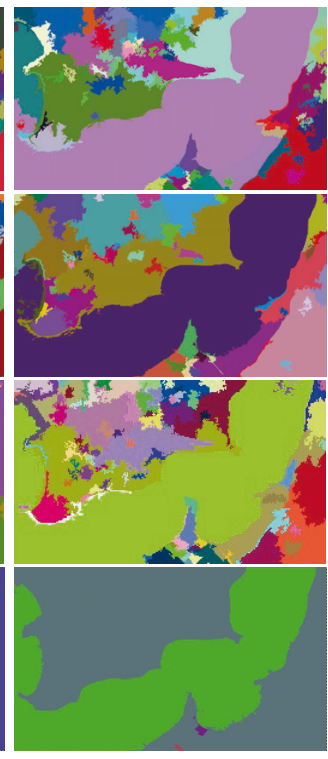

c)

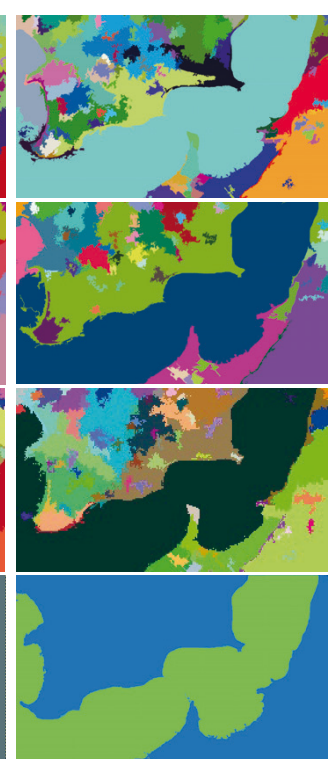

d)

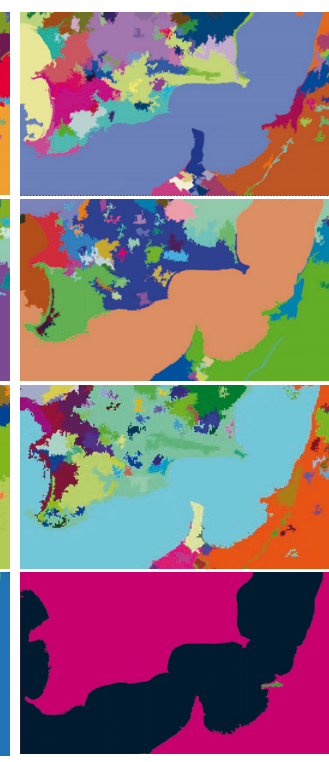

e)

Fig. 7. Application of segmentation for median filtered images for median filters of a) $3 \times 3$, b) $5 \times 5$, c) $7 \times 7$, d) $9 \times 9$, and e) $11 \times 11$. 
effect with a quick transformation and some blur. In this layer, the monochrome option was selected with values of $100 \%$ red, $200 \%$ green, and $-200 \%$ blue. To make a black and white infrared image, check the "monochrome" box. Since infrared film detects healthy, reflective vegetation (i.e., all the green stuff), it shows up bright. Slide the green
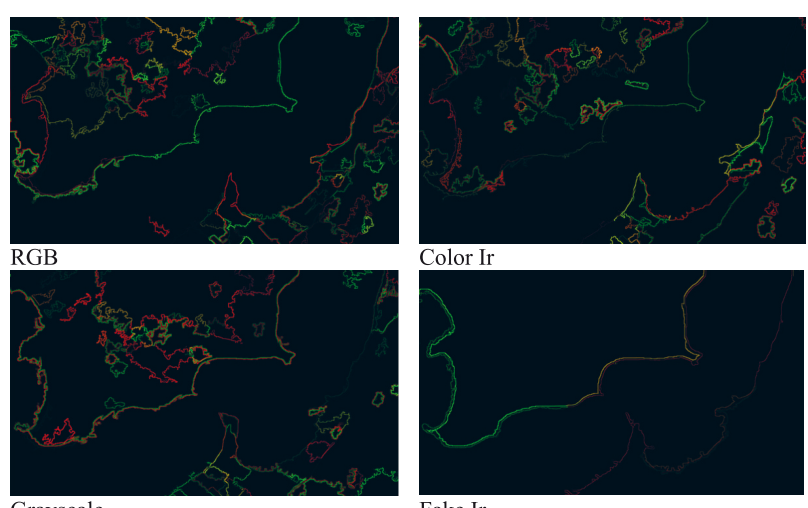

Grayscale

Fig. 8. Application of edge detection to the images after median filtering and segmentation with a $3 \times 3$ window.
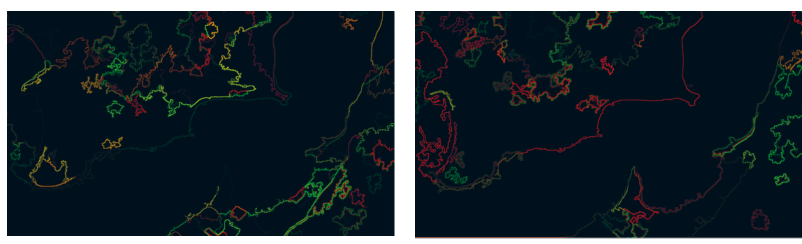

RGB
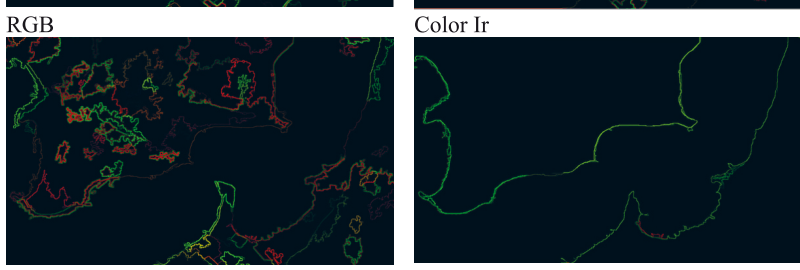

Fake Ir

Fig. 9. Application of edge detection to the images after median filtering and segmentation with a $5 \times 5$ window.
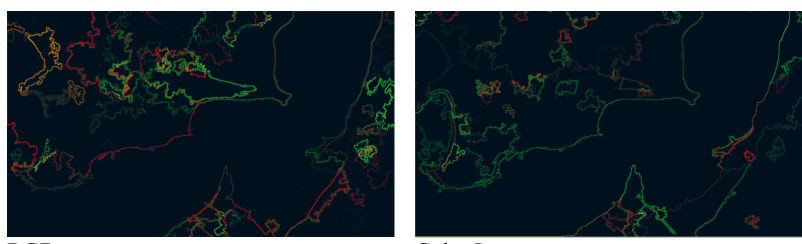

RGB
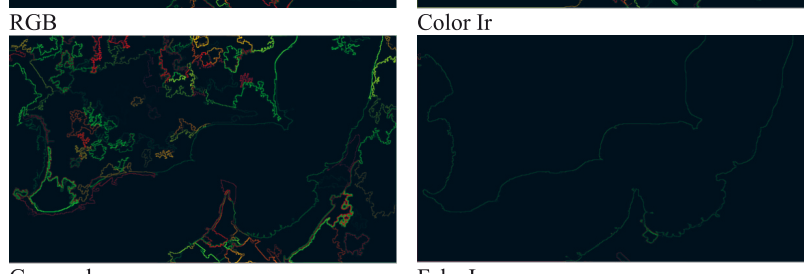

Fake Ir

Fig. 10. Application of edge detection to the images after median filtering and segmentation with a $7 \times 7$ window.

channel all the way to the right to pump that channel to $200 \%$. Kill the blue by moving its slider left to $-200 \%$. The opacity value of this image was set as $35 \%$ (Fig. 5). A noise value of $20 \%$ was applied to the newly added layer. After these operations we obtained the fake infrared image shown in Fig. 6-d. The RGB, color infrared, and grayscale images of the study area are shown in Fig. 6.

For all the images we applied several median filters of size $3 \times 3,5 \times 5,7 \times 7,9 \times 9$, and $11 \times 11$. The necessary software tools were developed within the study for the Median filtering by using the .NET Framework System.Drawing. dll library. In these software tools (which were developed in C\# programming language), the median pixel value is computed from the matrix of neighboring pixels in the source image and the new filtered image where the median values are assigned is saved as a new image. The median filtering is highly efficient if the resolution of the image needs to be preserved. This study aimed at minimizing the noise in the pixels representing the coastal zones where the land and sea meet. It was planned to improve automatic detection of the coastlines.

In the next step of the study, segments were produced from the median filtered images. For segmentation, a separate software tool was prepared in $\mathrm{C}++$. With
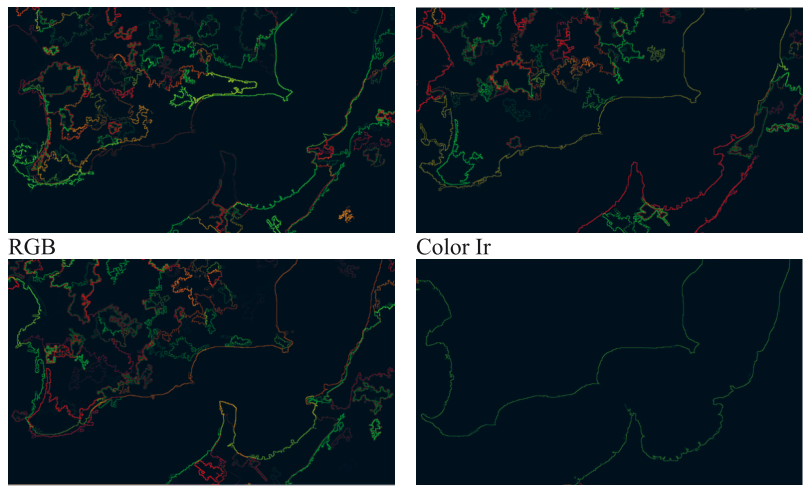

Grayscale

Fake Ir

Fig. 11. Application of edge detection to the images after median filtering and segmentation with a $9 \times 9$ window.
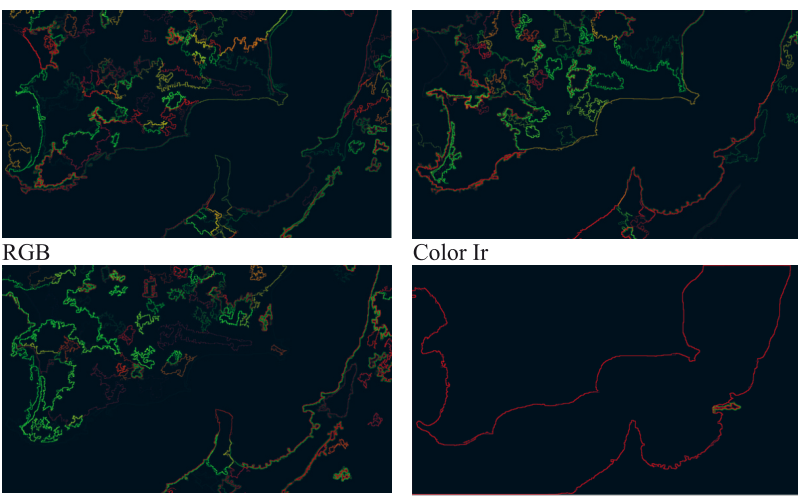

Grayscale

Fig. 12. Application of edge detection to the images after median filtering and segmentation with an $11 \times 11$ window. 


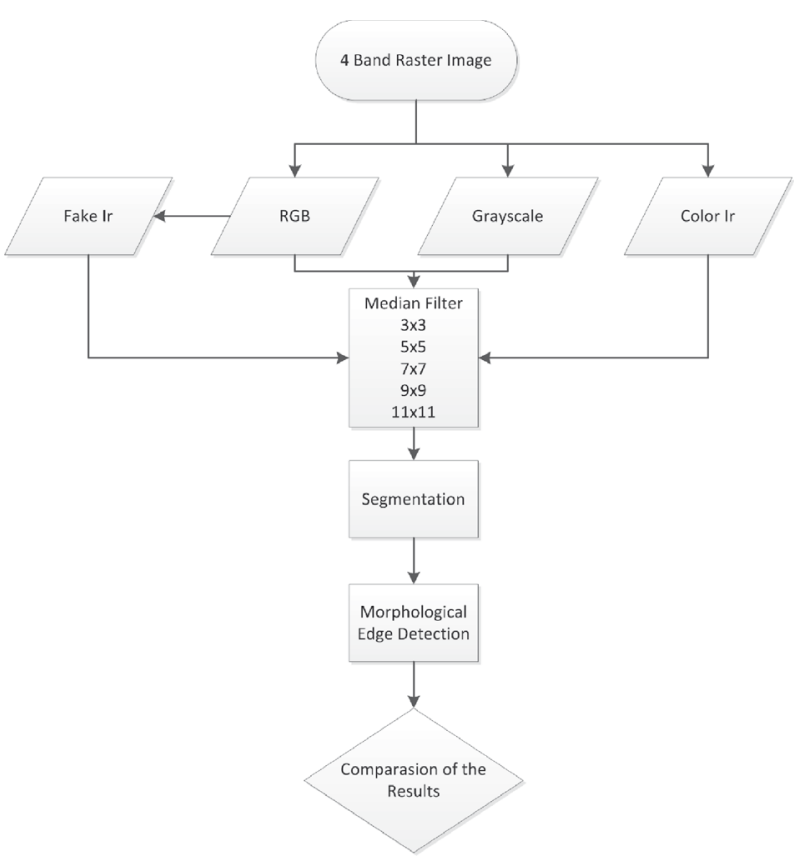

Fig. 13. Flow chart of the analysis.

this application, the pixels having similar values were determined and the corresponding image segments were formed. Therefore, the pixels representing the sea are identified and grouped. This segmentation was further used to determine the coastlines (Fig. 7).

In the last step of the study, a software tool in C\# was developed to apply morphological edge detection to the coastline images that were segmented in the previous step. Similar to the median filtering application, the .NET Framework System.Drawing.dll library was used in developing the morphological edge detection algorithm. The results of the study are shown in Figs 8-12. The complete flow chart of the analysis steps is shown in Fig. 13.

\section{Results and Discussion}

Since the results obtained for the median filtered and segmented fake infrared images with different window sizes were found to be more efficient to group sea pixels, these images were preferred to detect coastlines. The obtained results are given in Table 1. Using the results obtained with these images, we propose the optimal approach to automatic detection of coastlines.

As seen in Table 1, detection of coastlines with a 9x9 median filtering produces the closest results to manual digitization. No significant difference was observed in the process time for different window lengths. Considering the processing time, the time required for larger windows sizes increases but it is considered insignificant in automatic feature extraction.

The use of fake infrared images for automatic feature extraction, in particular, is one of the authentic contributions of this study. The developed processing algorithm was found to be highly efficient in the study region of $2 \times 2 \mathrm{~km}$, and we feel that similar results can be obtained in larger areas.

\section{Conclusion}

Important features like buildings and roads are manually detected by operators from infrared aerial photos. The developments in computer technology and digital image processing enable automatization of those processes and speed up the whole procedure. However, obtaining infrared images is not possible all the time. While many data providers offer RGB data free of charge, they have a different policy for infrared images. In this respect, the manipulation of RGB images as infrared images is possible thanks to image-processing technologies.

The infrared images obtained through manipulation of RGB images have proven to be efficient and the advantages of infrared images can be accessed easily. It is also considered that the proposed algorithm can be

Table 1. The results of automatic detection of the coastlines with fake infrared images.

\begin{tabular}{|c|c|c|c|c|}
\hline Method of Coastline Detection & $\begin{array}{l}\text { Coastline Length } \\
(\mathrm{km})\end{array}$ & \multirow{2}{*}{$\begin{array}{l}\text { Difference } \\
\quad(\mathrm{km})\end{array}$} & \multirow{2}{*}{ Ratio (\%) } & \multirow{2}{*}{$\begin{array}{l}\text { Total Processing } \\
\text { Time }\end{array}$} \\
\hline Manual Digitizing & 5.444918 & & & \\
\hline Edge detection after $3 \times 3$ median filtering and segmentation & 5.233843 & 0.251075 & 4.83 & $5,26 ”$ \\
\hline Edge detection after $5 \times 5$ median filtering and segmentation & 5.221063 & 0.223855 & 4.29 & 7 ' $14^{\prime \prime}$ \\
\hline Edge detection after $7 \times 7$ median filtering and segmentation & 5.310605 & 0.134313 & 2.53 & $8{ }^{\prime} 21 ”$ \\
\hline Edge detection after 9x9 median filtering and segmentation & 5.398489 & 0.046429 & 0.86 & 8' $42 ”$ \\
\hline Edge detection after $11 \times 11$ median filtering and segmentation & 5.370722 & 0.074196 & 1.38 & $9^{\prime} 29^{\prime \prime}$ \\
\hline
\end{tabular}


used in photogrammetric mapping. With improvements in software, features such as coasts, lakes, and rivers can be automatically detected with the proposed algorithm.

The software tools given in this study are authentic, independent, open source, practical, and open for further improvement. The quality of the images and their contrast and noise ratios have an impact on the proposed algorithm.

The surface properties and the contrast of the line features also affect the efficiency of the algorithm. To improve the efficiency of the given algorithms, several image enhancement methods such as increasing contrast and decreasing noise were also employed. Such further enhancements have proven to be useful in increasing the efficiency of the developed algorithm.

In summary, the RGB images were manipulated to produce fake infrared images and the coastline were automatically detected by using the developed algorithm, which proves to be the most efficient, and all the image processing was carried out by the developed software tools given in the appendices.

\section{Acknowledgements}

The authors would like to thank our reviewer for his/ her review, suggestions, and contributions.

\section{References}

1. SESLİ F.A. Mapping and Monitoring Temporal Changes for Coastal Region of Samsun, International Journal of the Physical Sciences, 5 (10), 1567, 2010.

2. PAPAKONSTANTINOU A., KOSTANTINOS T., PAVLOGEORGATOS G. Coastline change detection using UAV Remote Sensing GIS and 3D reconstruction, Planning and Economics (CEMEPE 2015) and SECOTOX Conference, 2015.

3. ZHANG H.G., LI D.L., SHI A.Q. On Scale Correction Model of Coastline and its Application for Coastline Remote Sensing Monitoring, Applied Mechanics and Materials, 303306, 734, 2013.

4. KANKARAA R.S., CHENTHAMIL SELVANA S., VIPIN J. MARKOSEA, B. RAJANA, AROCKIARAJA S. Estimation of long and short term shoreline changes along Andhra Pradesh coast using Remote Sensing and GIS techniques, Procedia Engineering 116, 855, 2015.

5. SHI X., CHEN H., QUI T., ZHANG Y. A Novel Coastline Detection Method of Remote Sensing Imagery with Local Gradient Based on Hybrid Active Contour Model, ICIC EXPRESS LETTERS 9 (6), 1651, 2015.

6. ZHANG Y., XUEMINIG L., JIANLI Z., DERUI S. A Study on Coastline Extraction and Its Trend Based on Remote Sensing Image Data Mining, Hindawi Publishing Corporation Abstract and Applied Analysis, Volume: 2013, Article ID:693194, 2013.

7. OZTURK D., SESLI F.A. Determination of Temporal Changes in the Sinuosity and Braiding Characteristics of the Kizilirmak River, Turkey. Pol. J. Environ. Stud. 24 (5), 2095, 2015.
8. EUROPEAN COMMISSION (EC) Towards a European Integrated Coastal Zone Management (ICZM) Strategy, Directorates General Environment, Nuclear Safety and Civil Protection; Fisheries, Regional policies and Cohesion, Brussels, 1999.

9. GESAMP (IMO/FAO/UNSECO-IOC/WMO/WHO/IAEA/ UNEP) Joint Group of Experts on the Scientific Aspects of Marine Environmental Protection, The Contribution of Science Integrated Coastal Management, GESAMP Reports and studies. No:61., 1996.

10. NORMAN B.J. Integrated Coastal Management to Sustainable Coastal Planning, PhD Thesis, RMIT University, Social Science and Planning College of Design and Social Context, 2010.

11. QIDWAI U., CHEN C.H. Digital Image Processing: an Algorithmic Approach with MATLAB. CRC Press, 2010.

12. WANG C., ZHANG J., MA Y. Coastal Land Covers Classification of High-Resolution Images Based on Dempster-Shafer Evidence Theory, International Conference on Computer Science and Software Engineering, Volume 1: Artificial Intelligence, December 12-14, 2008.

13. YANG J., PEIJUN L., YUHONG H. A multi-band approach to unsupervised scale parameter selection for multi-scale image segmentation, ISPRS Journal of Photogrammetry and Remote Sensing 94, 13, 2014.

14. ECOGNITION eCognition Developer 8.7 Reference Book, Trimble Documentation, München Germany, 438, 2011.

15. ALI M., CLAUSI D. Using the Canny Edge Detector for Feature Extraction and Enhancement of Remote Sensing Images, Geoscience and Remote Sensing Symposium, 5, 2298, 2001.

16. HONG N.T.K., CECILE B., TUAN V.P. Performance and Evaluation Sobel Edge Detection on Various Methodologies, International Journal of Electronics and Electrical Engineering, 2 (1), 15, 2014.

17. CHEN Y., DENG C., XIAXIA C. An Improved Canny Edge Detection Algorithm, International Journal of Hybrid Information Technology, 8 (10), 359, 2015.

18. KOENDERINK J.J. Theory of "Edge-Detection, Analysis for Science, Engineering and Beyond, Heidelberg: Springer Berlin, 35, 2012.

19. KARANDE K.J., TALBAR S.N. Laplacian of Gaussian Edge Detection for Face Recognition Using ICA, Independent Component Analysis of Edge Information for Face Recognition, 35, 2014.

20. SHIMA T., SAITO S., NAKAJIMA M. Design and Evaluation of More Accurate Gradient Operators on Hexagonal Lattices, IEEE Transactions on Software Engineering 32 (6), 961, 2010.

21. WEI G.W., JIA Y.Q. Synchronization Based Image Edge Detection, Europhysics Letters, 59 (6), 814, 2007.

22. PINNAMANENI B., RADHAKRISHNAN N., BHARATHI S. Mathematical Morphology Image Analysis Metrology, Machine Vision technology and Its Applications Workshop, 2013.

23. URL1 Wikipedia,http://en.wikipedia.org/wiki/Morphological_image processing 31 Jan 2016.

24. BELLAIRE G., TALMI K., OEZGUER E., KOSCHAN A. Characteristic Views: Obtaining 2-D Reconstructions From Color Edges, IEEE SSIAI'98, Tuscon USA, 1998. 